declared, Maria T. Terreri: None declared, Rolando Cimaz: None declared, Maria Katsikas: None declared, Dana Nemcova: None declared, Maria Jose Santos Speakers bureau: Novartis and Pfizer, Juergen Brunner Grant/research support from: Pfizer, Novartis, Consultant of: Pfizer, Novartis, Abbvie, Roche, BMS, Speakers bureau: Pfizer, Novartis, Abbvie, Roche, BMS, Mikhail Kostik: None declared, Kirsten Minden Consultant of: GlaxoSmithKline, Sanofi, Speakers bureau: Roche, Anjali Patwardhan: None declared, Kathryn Torok: None declared, Nicola Helmus: None declared

DOI: 10.1136/annrheumdis-2020-eular.2271

\section{FRI0467 DRUG SURVIVAL AND SAFETY OF BIOLOGICAL THERAPIES IN PATIENTS WITH JUVENILE IDIOPATHIC ARTHRITIS}

A. García Fernández ${ }^{1}$, A. Briones-Figueroa ${ }^{1}$, L. Calvo Sanz ${ }^{1}$, Á. AndreuSuárez ${ }^{1}$, J. Bachiller-Corral' ${ }^{1}$ A. Boteanu ${ }^{1} .{ }^{1}$ Hospital Ramón y Cajal, Rheumatology, Madrid, Spain

Background: Biological treatment (BT) has changed perspectives of JIA patients. Increasing data from real life experience have been reported.

Objectives: To compare drug survival, safety and efficacy of BT in patients with Juvenile Idiopathic Arthritis (JIA)

Methods: A retrospective observational study was conducted on JIA patients followed in a referal hospital and who had received at least one BT between 1999 and 2019.

Results: 218 BT in $130 \mathrm{JIA}$ patients were analyzed. $67.7 \%$ were women with a median age at diagnosis of 8 years old IQR (3-13) and a median age at the beginning of the BT of 15 years old IQR(7.8-21). $21.5 \%$ of the patients had uveitis during follow-up. BT were indicated due to: arthritis(73.9\%), uveitis(10.1\%), arthritis and uveitis(2.7\%), systemic activity(8.3\%) and macrophage activation syndrome (1,8\%). There were 130 BT started in 1st line, 55 in 2nd line, 20 in 3rd line, 10 in 4th line and 3 in 5 th line.

The 1st line BT most frequently indicated was Etarnecept(ETN) up to $40 \%$, followed by $30 \%$ Adalimumab(ADA) and $16,2 \%$ Infliximab(INF). The median duration of the 1 st line was 51 months IQR $(14-109,3)$. However, $53.8 \%$ of the 1st line BT were swiched: $28.3 \%$ due to adverse events, $25.7 \%$ due to 1 - failure and $25.7 \%$ due to $2 \circ$ failure. The BT that were discontinued were: INF (76.2\%) and Anakinra (ANAK) (75\%) due to adverse events and ETN $(59.6 \%)$ due to $1^{\circ}$ and $2^{\circ}$ failure. 55 patients started a 2 nd BT: $43.6 \%$ received ADA and $20 \%$ Tocilizumab (TCZ) with a median duration of 43 months IQR (12-90). 22 of 55 BT required a change: $75 \%$ of ETN and $59 \%$ of INF prescribed in 2 nd line were discotinued. The causes were: $40 \% 1^{\circ}$ failure, $28 \% 2^{\circ}$ failure and $12 \%$ remission. In 1 st line $87,6 \%$ of patients received TNF inhibitors, $74 \%$ mantained the target in 2nd line. In 3rd line TCZ was the most frequent BT. $71.5 \%$ of patients continue on BT. BT was withdrawn in 20 of 130 patients due to remission (40\%), adverse events $(30 \%)$, and pregnancy (10\%).

In the analysis by decades, 80 BT (36.7\%) were started from 1999 to 2008 and 138 BT (63.3\%) from 2009 to 2019. In the 1st decade ETN and INF were the most frequently prescribed and in the 2nd decade, ADA and TCZ $(p<0.0001)$. The 1 st BT in the 2 nd decade were indicated sooner compared to the 1st decade (1st decade: mean 119.5months $\operatorname{SD}(109.2)$; 2nd decade: mean 53.9 months SD(99.7); $p<0.0001)$. In 1st line BT, the BT prescribed in the 2 nd decade had a shorter duration than those in the 1st decade (1st decade: mean 84.1 months SD(71.8); 2nd decade: mean 51.7 months SD(5); $\mathrm{p}<0.0001)$.

In the survival analysis, TCZ and ADA were the BT with the highest survival $(p=0.001)$. Of the 31 patients that started TCZ, 61.3\% continue on TCZ, with a median duration of 46 month IQR(25-99) and $36 / 68(52,9 \%)$ still on ADA with a median duration of 61,5 month IQR(30.5-98).

Conclusion: $42.3 \%$ of patients required more than one BT. Since the onset of the BT there has been a change in prescription, probably related to the emerge of new targets and the evidence provided by clinical trials and guidelines. TCZ and ADA were the BT with the highest survival rate. On the other hand, INF and ANAK were the ones with the lowest survival rate. The most common causes of BT change in 1st line were adverse events in relation to INF and ANAK. In 2nd line there was a high rate of change in those patients who maintained TNFi, related to 1 - failure.

Disclosure of Interests: None declared

DOI: 10.1136/annrheumdis-2020-eular.4655

\begin{tabular}{|l|l}
\hline FRI0468 & SYSTEMIC AND CUTANEOUS POLYARTERITIS \\
& NODOSA IN COLOMBIAN PEDIATRIC PATIENTS: \\
& CUTANEOUS POLYARTERITIS IS NOT SO BENIGN
\end{tabular}

A. Medina ${ }^{1}$, M. Calle ${ }^{2}$, R. Eraso ${ }^{1,3,4}$, L. Hernandez ${ }^{1,4,5}$, J. S. Peinado Acevedo ${ }^{5}$, M. Velasquez ${ }^{6}$, A. Vanegas Va,5, $^{4, \text { D. Jaramillo Arroyave }}{ }^{4,5,7,8}$, C. Muñoz $z^{4,5,7,8}$.
${ }^{1}$ Universidad de Antioquia - Departamento de Pediatria, Medellin, Colombia; ${ }^{2}$ Universidad de Antioquia - Grupo de Estudio de Vasculitis Sistémicas, Medellin, Colombia; ${ }^{3}$ Hospital Pablo Tobón Uribe, Medellin, Colombia; ${ }^{4}$ Universidad de Anitoquia - Grupo de Reumatología, Facultad de Medicina - Departamento de Medicina Interna, Medellin, Colombia; ${ }^{5}$ Universidad de Antioquia - Grupo de Estudio de Vasculitis Sistémicas, Medellin, Colombia; ${ }^{6}$ Hospital Universitario San Vicente Fundacion, Hospital Infantil, Medellin, Colombia; ${ }^{7}$ Hospital Universitario San Vicente Fundacion, Medellin, Colombia; ${ }^{8}$ IPS Universitaria - Servicios de Salud - Universidad de Antioquia, Medellin, Colombia

Background: Polyarteritis nodosa (PAN) is the third most frequent vasculitis in pediatrics, Cutaneous PAN (CPAN) being more common that Systemic PAN (SPAN). CPAN is frequently described as a benign disease. In children, PAN onset is frequent between 9 and 11 years of age, with no sex differences, and its clinical features may be nonspecific.

Objectives: To characterize pediatric patients who were diagnosed with CPAN and SPAN and to compare their clinical features, treatments, and outcome.

Methods: A descriptive study was conducted in two centers from Medellin- Colombia, using retrospective data from January 2010 to December 2019. Patients under 18 years of age classified as PAN according to EULAR/PRINTO/ PRES(1) criteria were included. CPAN patients were defined according to EULAR/PRES definition (2). Data from medical records were registered, and were expressed in median and ranges and mean and standard deviation (SD) according to their distribution. A univariate analysis was carried out by comparing signs, symptoms, and treatment between CPAN and SPAN, and a $p$-value $<0,05$ was considered as significant.

Results: Twenty patients were included. The median age at diagnosis was ten years. $60 \%$ were boys. The median follow-up period was 27 months. CPAN was diagnosed in $11(55 \%)$ and SPAN in 9 patients (45\%). The most frequent symptoms were cutaneous manifestations (95\%), fever $(60 \%)$ and Calf Pain $(55 \%)$. Mucosal ulcers were described in four patients; 3 of them were defined as CPAN. Lingual necrosis was present in two CPAN, and peripheral nervous system involvement was found in one SPAN and two CPAN patients in skin affected with lesions; even though, no significant statistical differences between CPAN and SPAN were found in constitutional, cutaneous, muscle-skeletal symptoms, and acute phase reactants. Arteriographic anomalies as hepatic and renal microaneurysms, carotidal aneurysms without aortic involvement, and renal infarction were found in one patient each. Skin Biopsy was performed in 18 patients, being compatible with PAN in 16. All PAN patients (CPAN and SPAN) required treatment with glucocorticoids. None of the patients died during the follow-up period.

Conclusion: In this Colombian pediatric cohort of PAN patients, the disease was more common in boys than girls, and CPAN was more frequent than SPAN, as already been described. As is evident in this cohort, although CPAN has been considered a benign disease, these patients may be severely ill, requiring glucocorticoid treatment. Pediatric CPAN patients should be strictly followed with particular attention to identify systemic involvement, considering that constitutional, cutaneous, and muscle-skeletal features may be very similar between CPAN and SPAN.

References:

[1] Ozen S, Pistorio A, lusan S, et al. EULAR/PRINTO/PRES criteria for Henoch-Schönlein purpura, childhood polyarteritis nodosa, childhood Wegener granulomatosis and childhood Takayasu arteritis: Ankara 2008. Part II: Final classification criteria. Ann Rheum Dis. 2010 May;69(5):798-806

[2] Ozen S, Ruperto N, Dillon M, et al. EULAR/PReS endorsed consensus criteria for the classification of childhood vasculitides. Ann Rheum Dis. 2006;65(7):936-41.

Disclosure of Interests: None declared

DOI: 10.1136/annrheumdis-2020-eular.6512

\section{FRI0469 ACTIVE INFECTION IN PEDIATRIC SYSTEMIC LUPUS ERYTHEMATOSUS (SLE)}

K. L. Luo ${ }^{1}$, J. H. Lee ${ }^{2}$, B. L. Chiang ${ }^{2}{ }^{1}$ National Taiwan University Children's Hospital, Taipei, Taiwan, Republic of China; ${ }^{2}$ National Taiwan University Children's Hospital, Taipei, Taiwan, Republic of China

Background: SLE is the autoimmune disease involving multiple systems Infections might mimic SLE flare, leading to confusion over the diagnosis and appropriate treatment. To distinguishing acute infection from active flare always remains a clinical challenge.

Objectives: We aim to explore the potential parameters in identifying active infection and disease activity in pediatric SLE. 